\title{
A Thermal Physiological Comparison of Two HazMat Protective Ensembles With and Without Active Convective Cooling
}

\author{
Rebecca Williamson, M.S. \\ Jorge Carbo, B.S. \\ Bernadette Luna, M.S.M.E. \\ Bruce W. Webbon, Ph.D.
}

\begin{abstract}
Wearing impermeable garments for hazardous materials clean up can often present a health and safety problem for the wearer. Even short duration clean up activities can produce heat stress injuries in hazardous materials workers. It was hypothesized that an intemal cooling system might increase worker productivity and decrease likelihood of heat stress injuries in typical HazMat operations.
\end{abstract}

Two HazMat protective ensembles were compared during treadmill exercise. The different ensembles were created using two different suits: a Trelleborg VPS suit representative of current HazMat suits and a prototype suit developed by NASA engineers. The two life support systems used were a current technology Interspiro Spirolite breathing apparatus and a liquid air breathing system that also provided convective cooling. Twelve local members of a HazMat team served as test subjects. They were fully instrumented to allow a complete physiological comparison of their thermal responses to the different ensembles. Results showed 
that cooling from the liquid air system significantly decreased thermal stress. The results of the subjective evaluations of new design features in the prototype suit were also highly favorable. Incorporation of these new design features could lead to significant operational advantages in the future.

\section{Introduction}

The manufacture, transportation, and use of hazardous materials have become an accepted part of modem technology around the world. In order to keep workers safe when exposed to hazardous substances, some form of protective equipment is necessary. Many toxic materials require full body protection due to the caustic nature of the vapors/chemicals involved. Wearing a fully enclosed, vapor/chemical protective suit with a breathing system creates new risks for the user.

It is a well established fact that the use of protective clothing and respiratory equipment decreases a worker's ability to perform his job (1). In general, simply wearing a heavy self-contained breathing apparatus (SCBA) can reduce the working time to exhaustion and the maximal work pace by $13-20 \%(2,3,4)$. Increases in blood pressure, respiratory minute ventilation, and heart rate have been observed while wearing protective clothing $(5,6)$. Moving the weight of the SCBA and the "hobbling effect" of the clothing have been found to increase energy expenditure, resulting in a greater total body heat load $(1,7,8)$. Wearing protective clothing and a respirator ensemble is potentially dangerous since they can pose significant thermoregulatory and cardiovascular stress to the wearer, even at low to moderate work intensities and in a thermally neutral environment. The stress is greatly increased in a hot ambient environment. 
Despite many studies illustrating heat stress problems caused by protective clothing, little has been done to decrease the degree of heat stress experienced in these suits besides instituting work breaks and time limits in suits. The present study was designed to answer questions regarding the energy cost and heat stress when ambulating in a Level A HazMat protective ensemble. The first question to be investigated was to determine if the liquid air breathing system with convective cooling developed by NASA was effective in decreasing the heat stress experienced by hazardous materials workers while in Level A suits. During an earlier program . a prototype HazMat suit was developed at NASA Ames Research Center in an effort to integrate space suit design concepts into HazMat suits to determine if operational efficiency and comfort could be achieved. Therefore, another research question was to ascertain whether the Ames suit offered any advantages over the currently used equipment with regard to subjective comfort, operations, and thermal stress. 


\section{Methods}

Subjects

Twelve male volunteers, ranging in age from 31 to 47 years of age (mean $38.3 \pm 4.3$ years) served as test subjects. The physical characteristics (mean $\pm S D$ ) of the subjects were height $182 \pm 5.3 \mathrm{~cm}$, weight $89.5 \pm 10.4 \mathrm{~kg}$, body fat 18.1 $\pm 3.0 \%$, and maximal oxygen uptake $52.4 \pm 6.8 \mathrm{ml} / \mathrm{kg}$ per minute. All subjects were local firefighters who had prior experience and training in Level A HazMat ensembles. All subjects underwent annual physicals through the Fire Department prior to the study. Before beginning the experiments, all subjects underwent a treadmill test to exhaustion utilizing the Bruce treadmill protocol in order to determine maximal oxygen uptake.

\section{Experimental Design and Exercise Protocol}

All subjects performed an identical work profile on a motor driven treadmill in four different clothing/breathing ensembles. The continuous work profile (see Table 1) consisted of a 5 min control period standing still on the treadmill, a $3 \mathrm{~min}$ walking warm up at $2.6 \mathrm{~km} / \mathrm{h}$, followed by a $45 \min$ period of walking at $3.2 \mathrm{~km} / \mathrm{h}$, a $3 \mathrm{~min}$ cool down at $2.6 \mathrm{~km} / \mathrm{h}$, and a final control period of standing still for 5 min. The treadmill remained at $0 \%$ grade throughout the entire work profile. This exercise level was picked to be representative of actual HazMat field situations.

The first round of tests served as a control (C) in which the subjects were dressed in t-shirts, shorts, and running shoes. The second round of tests (Tborg) utilized a standard Level A HazMat vapor protective suit with a self contained breathing apparatus (SCBA). The third round of tests (T/cryo) utilized the same 
protective suit with a cryogenic, liquid air backpack in place of the SCBA. The final round of experiments ( $\mathrm{A} / \mathrm{cryo}$ ) utilized the NASA-Ames prototype Level A HazMat suit and the cryogenic backpack. Only five subjects performed this last round of experiments due to the size constraints of the prototype suit. All exercise sessions took place in an environmentally controlled chamber which was kept at $24.4^{\circ} \mathrm{C}, 40-45 \% \mathrm{RH}$, with an air speed of approximately $3540 \mathrm{~V} / \mathrm{min}$ (125 scfm).

\section{Protective Ensembles}

A Trelleborg Trellchem vapor protective suit (VPS) was used in two sets of trials (Tborg and T/cryo). The Trellchem suit is an NFPA 1991 Level A vapor protective suit with a single layer construction. It utilizes an SCBA which can operate independently (no umbilical) or in conjunction with an external gas supply with a through-suit penetrator. Access into this suit is through a gas-tight zipper which zips vertically from the head cavity to the lower torso. The test subjects used standard butyl rubber boots and gloves. All breathing gas was expelled into the suit and collected at exhaust valves on the head cavity of the suit. The valve housings were modified to facilitate the collection of expired gases for analysis. This expired gas pressurized the suit to a gage pressure of $-5.1 \mathrm{~cm}(2 \mathrm{in})$ of water.

The Trelleborg suit was used in the Tborg runs using an Interspiro Spirolite High Performance Breathing Apparatus (HPBA). The weight of the complete protective ensemble was $19 \mathrm{~kg}$. The same suit was also used in the third round (T/cryo) tests with an Arrowhead Cryogenic Environmental Control Unit (ECU) which provides both breathing air and convective cooling. This was originally 
developed for NASA for use by rocket fueling crews and in other hazardous work environments. An air circulating manifold was installed in this suit. An air-tight aluminum penetrator plate with electro-cardiogram (ECG) capabilities, core temperature (rectal) and skin temperature electrical pass-throughs was installed on the right side of the VPS suit. Both large and extra-large VPS suits were used. The suit was leak-tested on a routine basis. The weight of the complete ensemble for the T/cryo tests was $35.2 \mathrm{~kg}$.

A prototype HazMat suit developed at NASA Ames Research Center was used in the A/cryo tests. The Ames HazMat suit is a prototype Level A suit intended for realistic concept feasibility and operational demonstrations in nonhazardous environments. This suit incorporates several design features which are direct spin-offs from space suits including a rigid, hemispherical visor, and a rearentry hatch system incorporating the Arrowhead ECU life support. Unique to this suit is a load-bearing system which utilizes a commercially available backpack hamess and waist belt attached to a rigid frame which locks into suit lock rings. All of the suit weight, including the weight of the life support system, is transferred via this frame to the shoulders and hips of the suit subject. The back of the suit is a hard shell designed to be used with a suit lock system attached to a HazMat vehicle. This suit lock system consists of a series of concentric rings and shells, both on the suit and vehicle, which allows for the transfer of a suited subject from a vehicle into the suit without cross-contamination. The suit can also be used in a manual mode without the docking station as was done for these tests.

Another unique characteristic of the Ames suit is the double layer construction. The inner layer acts as a pressure barrier while the outer layer is the 
main chemical barrier. The double layer design eliminates the necessity for the suit subject to wear a positive pressure mask because air from the cryogenic ECU is recirculated through the suit providing fresh breathing air as well as cooling. Another feature of the Ames suit is the use of quick disconnects for the gloves and boots. This allows for immediate resizing and selection of boots and gloves which are appropriate for the chemical threat. The Ames suit also operates at a pressure of $-5.1 \mathrm{~cm}(2 \mathrm{in}$.) of water and has three exhaust valves located on the chest of each layer of the suit. The outer layer valves were removed and newly designed valves with hose connectors were installed to collect expired gases from the inner layer. An air-tight aluminum penetrator plate incorporating ECG, core temperature and skin temperature electrical pass-throughs was installed on the right side of the inner layer of the suit. The suit was also leak-tested on a routine basis.

The total weight of the $\mathrm{A} / \mathrm{cryo}$ protective ensemble was $36.5 \mathrm{~kg}$. This includes the weight of the liquid air, $\sim 5.9 \mathrm{~kg}$, which is evaporated and exhausted during operation. The Ames suit was designed to be used in conjunction with a special, sealed HazMat vehicle. The suit contains a substantial amount of weight due to the hardware which enables"docking" with the vehicle in a hazardous environment and the suit occupant to "lock" directly into the vehicle interior without being exposed to the ambient environment. If such a vehicle was not available, as would be the case for most local HazMat situations, then the suit design could be simplified considerably and the weight reduced to approximately the same as the Trelleborg VPS.

\section{Life Support System}


An Interspiro Spirolite SCBA/HPBA was used in the Tborg test runs. The HPBA combines a positive pressure mask and a 4500 psi gas cylinder with a 45 minute capacity. This system weighs approximately $12.27 \mathrm{~kg}$ and is carried using a shoulder harness. During the Tborg runs the HPBA unit was worn by the test subjects, but the breathing air was supplied from an extemal tank using an umbilical hose and through-suit connector. The umbilical allows the suited subject to remain in the suit for more time than a backpack cylinder would allow. The tests were approximately one hour in duration with an additional 15 minute for a margin of safety. This time exceeds the capability of currently available backpack cylinders. This system was chosen because it is currently used by a large segment of the HazMat community including the Santa Clara County Fire Protection District. The gas cylinders were filled using a high-pressure breathing air compressor.

The Arrowhead cryogenic ECU was used for the T/cryo and A/cryo test runs. The ECU was developed for NASA HazMat teams working in hot environments, such as NASA Kennedy Space Center in Florida. The ECU provides both breathing air and convective cooling by vaporizing cryogenic or liquid air stored in an insulated dewar within the backpack. The dewar operates at a pressure less than $1,137 \mathrm{kPa}$ (165 psi). Cryogenic air is vaporized at a rate of $\sim 43$ $\mathrm{V} / \mathrm{min}(1.5 \mathrm{acfm})$ which is ejected into a Venturi tube. This injection of new gas mixes with $382 \mathrm{~V} / \mathrm{min}(13.5 \mathrm{acfm})$ of suit air inducted into the Venturi tube. It is then vented through a heat exchanger and into the suit manifold. The heat exchanger regulates the vaporization of cryogen based on the temperature of the inducted suit air. As the suit subject produces more heat, warmer air passes through the heat exchanger resulting in the vaporization of more cryogen. The 
ECU dewar has a storage capacity of $5.9 \mathrm{~kg}$ ( $13 \mathrm{lbs}$ ) of liquid air. The entire ECU weighs a total of $15.9 \mathrm{~kg}$ ( $35 \mathrm{lbs})$ when full. The ECU has an off, low, medium and high setting for the air outlet into the suit manifold. The high setting was used for all test runs.

A manifold system was used to circulate air through both the Ames and standard HazMat suits. The manifold directs a majority of the ECU outlet air to a spray bar in the head cavity of the standard HazMat suit and to a similar spray bar in the Ames suit. The exhaust of both systems is designed to de-fog the visors and to wash the head cavity with fresh air thus preventing a carbon dioxide build-up in the oro-nasal area.

The ECU is designed to be carried on the back using shoulder straps and a waist belt. These straps concentrate the weight of the unit on the shoulders of the wearer which quickly becomes uncomfortable. A more comfortable hamess and waist belt which transfers most of the load to the subject's hips was developed for the Ames suit. This was used during the $\mathrm{A} /$ cryo test runs.

The ECU is normally refilled with cryogenic air using a large refill station that mixes liquid nitrogen and liquid oxygen in the correct proportions. Liquid air can not be stored for extended periods (days) because the component gasses boil off at different rates, thus changing the composition of the gas with storage time. It was recognized that this could cause a serious logistical problem which would have to be addressed and solved before the HazMat community could adopt liquid air ECU's. Therefore, the Extravehicular and Protective Systems Branch at Ames Research Center, in conjunction with Brigham Young University, designed and fabricated a small and very simple refill station which requires only liquid nitrogen 
and compressed air for operation. Both are relatively easy to acquire and can be stored for long periods. Thus a fire station could have these supplies on hand and quickly charge their liquid air ECU's on demand. This refill station uses a copper coil submerged in a bath of liquid nitrogen. Compressed breathing air is expanded to $\sim 689 \mathrm{kPa}(-100 \mathrm{psi})$ and then passed through the cold copper coil where it condenses. The condensed, now liquid, air is then transferred directly into the dewar. of the ECU. The oxygen concentration, peak flow rate, and weight of the ECU were measured and recorded before each of our tests.

\section{Physiological Measurements/Equipment Used}

A data acquisition system interfaced with a personal computer was utilized to acquire physiological data during the tests. All physiological responses were monitored continuously and logged once per second. In order to understand the additional physiological cost of ambulating in the various ensembles, there were specific data collection intervals that were of special importance. Intervals of interest were measurements that were recorded in the last minute of the standing control period (Stage 1) compared with the measurements of those same parameters taken at the last minute of exercise before cooling down (exercise minutes 53-54, Stage 3). Comparisons were then made between the different suit ensembles using measurements made during these intervals.

Heart rate was monitored using a Hewlett Packard 78352A three lead ECG monitor. Five skin temperatures were measured at the chest, upper arm, lower arm, upper leg and lower leg with thermistors. Mean skin temperature was calculated by adapting an equation by Hardy and DuBois (9) 
The equation is

$$
\begin{gathered}
\mathrm{T}_{\text {skin }}=.2\left(\mathrm{~T}_{\text {lower leg }}\right)+.19\left(\mathrm{~T}_{\text {upper leg }}\right)+.13\left(\mathrm{~T}_{\text {lower arm }}\right) \\
+.06\left(\mathrm{~T}_{\text {upper } \mathrm{arm}}\right)+.42\left(\mathrm{~T}_{\text {chest }}\right)
\end{gathered}
$$

Rectal temperature was measured with series 400 thermistors from YSI inserted 10 cm. ( 4 in.) beyond the anal sphincter. Ear canal temperature was measured before and immediately after each test with an infrared ear canal thermometer (Thermoscan). Expired air flow rate was measured using a pneumotach (Model No. 4813 from Hans Rudolph Company). Gas concentrations of expired air were analyzed with a $C D$ 3A carbon dioxide analyzer and an S-3A/1 oxygen analyzer from Appliued Electrochemistry. Expired air was collected using a mouth piece/nose clip system in the $C$ (unsuited control) tests and through the exhaust manifolds in the suited trials as described earlier in this paper. Subjects nude weight was measured and recorded before and immediately after all tests using a platform scale.

\section{Subjective Measurements}

Two subjective measurements, ratings of perceived thermal comfort and perceived exertion level, were taken at several intervals during the tests (Tables 2 and 3). Subjects were questioned at $5 \mathrm{~min}, 20 \mathrm{~min}$, and $40 \mathrm{~min}$ after the start of the $3.2 \mathrm{~km} / \mathrm{h}$ walk (Stage 3) portion of each test. Subjects gave the test director hand gestures to indicate their subjective ratings on each scale while they walked on the treadmill.

\section{Results}


Oxygen Cost of Exercise

Figure 1 illustrates the mean oxygen consumption rate during the control period and during the last minute of Stage 3 (walking at $3.2 \mathrm{~km} / \mathrm{h}$ ) of the exercise protocol in all four suit ensembles. The mean oxygen consumption rate during this last minute of Stage 3 was an average of $180 \%, 290 \%, 157 \%$ and $175 \%$ over the mean of the standing control period (Stage 1) in the C, Tborg, T/cryo and Acryo runs, respectively. Ambulating in the Tborg test runs without cooling produced an average increase in oxygen consumption of $\sim 0.41 / \mathrm{min}$ compared to the unsuited $\mathrm{C}$ runs, a $43 \%$ increase over ambulating in shirt sleeves. The runs with the cooling system in the T/cryo test runs produced an average increase of only $\sim 0.09 \mathrm{l} / \mathrm{min}$ over ambulating in the unsuited control condition, an increase of only $9.7 \%$. The oxygen cost of walking in the NASA suit was $\sim 0.14 \mathrm{1} / \mathrm{min}$ above ambulating in the unsuited control condition, an increase of $15.2 \%$.

The statistical means of the $5 \mathrm{~min}$ control period were $0.33( \pm 0.05 \mathrm{~V} / \mathrm{min})$, $0.36( \pm 0.10 \mathrm{l} / \mathrm{min}), 0.39( \pm 0.06 \mathrm{l} / \mathrm{min})$ and $0.39( \pm 0.07 \mathrm{l} / \mathrm{min})$ for the $C$, Tborg, $\mathrm{T} / \mathrm{cryo}$, and $\mathrm{A} / \mathrm{cry}$ o runs respectively. These means of the control period were not found to be significantly different between the $C$ and Tborg runs, or between the $C$ and $A$ cryo runs. These means were significantly different $(p=.01)$ between the $C$ and the T/cryo runs.

\section{Rectal Temperature}

Figure 2 illustrates the mean rectal temperature during the control period and the last minute of exercise in all test conditions. The mean core temperature rose to $37.2 \pm 0.3^{\circ} \mathrm{C}, 38.0 \pm 0.3^{\circ} \mathrm{C}, 37.4 \pm 0.4^{\circ} \mathrm{CF}$, and $37.7 \pm 0.2^{\circ} \mathrm{C}$ in the $\mathrm{C}$, Tborg, 
$\mathrm{T} / \mathrm{cryo}$, and $\mathrm{A} / \mathrm{cryo}$ runs, respectively. This represented an average rectal temperature increase across subjects of $0.18 \%, 1.2 \%, 0.4 \%$, and $0.8 \%$ for the $\mathrm{C}$, Tborg, T/cryo, and A/cryo runs, respectively. The magnitude of change between the $C$ and Tborg runs at the last minute of exercise was $0.7^{\circ} \mathrm{C}$, a $1.8 \%$ increase over the $C$ values. The increases over the Stage 3 core temperatures in the $C$ runs were $0.2^{\circ} \mathrm{C}(0.3 \%)$ in the $\mathrm{T} / \mathrm{cryo}$ runs and $0.5^{\circ} \mathrm{C}(0.8 \%)$ in the $\mathrm{A} / \mathrm{cryo}$ runs. In the T/cryo runs; two subjects experienced a decrease in core temperature compared with their temperature during the $5 \mathrm{~min}$ control. Three subjects in the control runs also experienced a drop in core temperature from the beginning to the end of the trial. There was no statistically significant difference between the mean core temperatures in the control phase of any of the suit ensembles.

\section{Skin Temperatures}

Figure 3 provides the mean skin temperature during the exercise protocols in all test types. The mean skin temperature across subjects at the last minute of exercise was $31.8 \pm 0.8^{\circ} \mathrm{C}, 37.0 \pm 0.7^{\circ} \mathrm{C}, 33.4 \pm 1.0^{\circ} \mathrm{C}$, and $36.0 \pm 0.2^{\circ} \mathrm{C}$ for the $\mathrm{C}$, Tborg, T/cryo, and A/cryo runs, respectively. This represented an average percentage increase of $-1.0 \%, 7.6 \%, 4.6 \%$, and $6.9 \%$, respectively, over the means of the control period for each run. The difference between the Stage 3 measurements was largest between the $C$ and Tborg runs, with an increase of $5.24^{\circ} \mathrm{C}$ over the $\mathrm{C}$ runs, an increase of $14.0 \%$. The increase over the $\mathrm{C}$ runs was $4.9 \%$ and $11.7 \%$ for the $T / c r y o$ and $A / c r y o$ runs, respectively.

Note: The mean skin temperature was lower during the last minute of exercise than during the control period in 5 subjects during the $\mathrm{C}$ runs. 


\section{Mean Body Temperature}

Figure 4 illustrates the mean body temperature response during the control periods of all runs and the last minute of Stage 3. Mean body temperature was calculated with the following equation (10):

$$
\mathrm{T}_{\text {body }}=0.67 \mathrm{~T}_{\text {rectal }}+0.33 \mathrm{~T}_{\text {skin }}
$$

The mean skin temperature across subjects during the last minute of exercise was $35.4 \pm 0.3^{\circ} \mathrm{C}, 37.6 \pm 0.3^{\circ} \mathrm{C}, 36.1 \pm 0.6^{\circ} \mathrm{C}$, and $37.2 \pm 0.1^{\circ} \mathrm{C}$ for the $\mathrm{C}$, Tborg, T/cryo and $\mathrm{A} /$ cryo runs respectively. This represented an average percentage increase of $0.3 \%, 2.6 \%, 1.0 \%$, and $2.0 \%$ increase, respectively, over the means of the control period. The largest difference at the last minute of Stage 3 was between the $C$ and Tborg runs, with a mean increase of $2.2^{\circ} \mathrm{C}$, a $4.1 \%$ increase over the last minute of Stage 3 in the $C$ runs. The T/cryo and $A / c r y o$ runs elicited smaller increases over the $\mathrm{C}$ runs with the $\mathrm{T} / \mathrm{cryo}$ runs averaging $0.7^{\circ} \mathrm{C}(1.2 \%)$ and the $\mathrm{A} / \mathrm{cryo}$ runs averaging an increase of $1.8^{\circ} \mathrm{C}(3.3 \%)$. There was a statistically significant difference in the mean body temperature in the control periods amongst the runs except for the $\mathrm{C}$ run compared to the T/cryo run, in which there was no statistical difference in the means of the control period. 


\section{Heart Rate}

The mean heart rate at the last minute of exercise was 96 bpm $( \pm 18.5), 144$ bpm $( \pm 21), 100( \pm 16.4)$ and $121( \pm 15.6)$ in the $C$, Tborg, T/cryo, and $A / c r y o$ runs, respectively. The magnitude of change over the $\mathrm{C}$ runs at the last minute of Stage 3 was $50.5 \%, 4.4 \%$, and $26.3 \%$ for the Tborg, $\mathrm{T} / \mathrm{cryo}$ and $\mathrm{A} / \mathrm{cryo}$ runs, respectively.

Note: In the control phase, there were three subjects whose heart rates were lower at the last minute of exercise than they were in the last minute of the control period.

\section{Body Water Loss}

Figure 5 shows the mean body water losses across all tests. The increase in body water loss between the $C$ and Tborg runs represented a $249 \%$ increase, the increases between the $\mathrm{C}$ and $\mathrm{T} / \mathrm{cryo}$ runs averaged $37 \%$, and the increase between the $\mathrm{C}$ and the $\mathrm{A} / \mathrm{cryo}$ runs represented an average increase in body water loss of $72 \%$.

\section{Rating of Percieved Exertion}

Figure 6 illustrates the rating of perceived exertion at $5 \mathrm{~min}$ and at $40 \mathrm{~min}$ into Stage 3.

\section{Rating of Thermal Comifort}

Figure 7 illustrates the rating of percieved thermal comfort at $5 \mathrm{~min}$ and at $40 \mathrm{~min}$ into Stage 3. 


\section{Discussion}

Oxygen Cost

The resting (standing) oxygen consumption rate $(\sim 0.4 \mathrm{~V} / \mathrm{min})$ in full HazMat gear in this study agrees with a similar study for resting values (11) in chemical protective gear. The oxygen cost of walking unsuited at $3.2 \mathrm{k} / \mathrm{h}$ was found to be $0.92 \mathrm{~V} / \mathrm{min}$ in our study which is comparable to the $.97 \mathrm{~V} / \mathrm{min}$ found by Smolander and colleagues (12). The Trelleborg suit with the SCBA clearly adds a load to an individual's work load. Ambulating in the Trelleborg ensemble required an increase in energy output of $43 \%$ compared to ambulating at the same speed in shirt sleeves. Our average peak oxygen consumption of $1.32 \mathrm{l} / \mathrm{min}$ is also similar to other rešearchers' findings with similar clothing ensembles and protocols $(2,12$, 13). In the T/cryo run, adding the cryogenic backpack significantly decreased the oxygen consumption $(\mathrm{p}=.0002)$ at the last minute of exercise from the values seen in the Tborg runs. It should also be noted that the T/cryo suit ensemble was approximately $16 \mathrm{~kg}$ heavier than the Tborg set up, yet the oxygen cost of the exercise was still less by a factor of $23 \%$. The A/cryo trials also appeared to generate lower oxygen consumption levels at the last minute of exercise than the Tborg runs, however the difference in values was not statistically significant. The NASA - Ames prototype suit fit more snugly, as well as being heavier than the Trelleborg suit. It's presumable that the increased oxygen consumption over that seen in the Tborg runs could be due to the increased hobbling effect the smaller Ames suit caused on the larger subjects. Some subjects also felt a tightness in their chest due to the constriction of the prototype backpack mounting system. This could have also caused some of the difference seen in oxygen consumption values. 


\section{Rectal Temperature}

The highest rectal temperatures measured were during the Tborg runs. This expected result was caused by the lack of air circulation which would provide convective cooling inside the suit and the lack of significant evaporative cooling from the skin. The mean rectal temperature reached during the Tborg runs during the last minute of Stage 3 was $37.8^{\circ} \mathrm{C}$, which is comparable to other studies with similar clothing ensembles and protocols $(7,12)$. The rectal temperatures in the last minute of exercise for the T/cryo condition was $-37.38^{\circ} \mathrm{C}$, which was $.57^{\circ} \mathrm{C}$ lower than the $T /$ cryo runs and highly significant $(p=.0002)$ compared to the Tborg runs. The mean rectal temperature at the last minute of the $\mathrm{A} / \mathrm{cryo}$ runs was also lower than the mean rectal temperature in the Tborg runs, but the difference was not statistically significant. Again, this could be partly explained by the smaller prototype suit which offered less of an opportunity for evaporative cooling and less of an opportunity for cooled air to reach the extremities.

In the Tborg runs, skin and core temperatures never reached equilibrium, but continued to rise in the walking cool down and standing rest phases of the test. This phenomenon was also observed in several other studies $(1,7,14)$. In this study, in the runs with cooling (both $\mathrm{T} / \mathrm{cryo}$ and $\mathrm{A} / \mathrm{cryo}$ ), both skin and core temperatures equilibrated before the end of the exercise period and in some cases, decreased during the walking cool down and the standing rest periods due to adequate cooling of the skin. This indicates that the convective cooling produced by the cryogenic breathing system prevented the heat stress that occurs in enclosed suits over time. 
Safety rules for most local HazMat operations state that a core temperature increase of $0.8^{\circ} \mathrm{C}$ over initial entry level temperature will preclude an individual's re-entry into the emergency site (15). In this study, two individuals had core temperature increases of $0.8^{\circ} \mathrm{C}$ or more in the Tborg runs. In the T/cryo runs no one experienced a core temperature rise of more than $0.4^{\circ} \mathrm{C}$, and in the $\mathrm{A} / \mathrm{cryo}$ runs, the highest core temperature increase was $0.7^{\circ} \mathrm{C}$. It appears from these data that the effect of the cryogenic cooling is a highly positive one, in that it keeps the core temperature from rising to dangerous levels by increasing the amount of convective and evaporative cooling from the skin surface.

\section{Mean Skin Temperature Mean Body Temperature}

Also as expected, the highest mean skin temperature and mean body temperature were measured during the Tborg runs, which again, would be due in great part to the lack of convective and evaporative cooling in an enclosed suit. Peak skin temperatures measured in this study are higher than those reported by Smolander et al. (12). However the magnitude of the increase in temperature is somewhat similar; $2.2^{\circ} \mathrm{C}$ in Smolander et al, and $2.5^{\circ} \mathrm{C}$ in this study. When cooling was added, the mean skin temperature at the last minute of exercise dropped from $37.0^{\circ} \mathrm{C}$ in the Tborg runs to $33.4^{\circ} \mathrm{C}$ in the T/cryo runs, a difference which was highly statistically significant ( $p=.000003$ ). The $\mathrm{A} / \mathrm{cryo}$ ensemble also of fered a cooling benefit with mean skin temperatures at the last minute of exercise measuring $36.0^{\circ} \mathrm{C}$, although this was not statisically significant The mean body temperature at the last minute of exercise was $36.1^{\circ} \mathrm{C}$ in the $\mathrm{T} / \mathrm{cryo}$ runs, which was significantly $(\mathrm{P}=.0000007)$ different than in the Tborg runs. In the $\mathrm{A} / \mathrm{cryo}$ runs the mean body 
temperature at the last minute of exercise was $37.2^{\circ} \mathrm{C}$. This was $0.4^{\circ} \mathrm{C}$ lower than the uncooled suit run, but this difference did not reach statistical significance. These data indicate that the thermal stress on the subject was greatly decreased by the addition of the cryogenic cooling backpack, and that the additional weight of the backpack was not a deleterious factor.

Other researchers (16) have put forth the idea that the degree of convergence of skin temperature toward the rectal temperature indicates a decreasing tolerance time in encapsulated suits. The authors suggested that when the difference between mean skin and rectal temperature converges to $1^{\circ} \mathrm{C}$ or less, the subject is on the verge of hyperthermia, and they should be removed from the hot environment or stop working. Such body temperature convergence indicates there is very little potential for internal heat transfer from the body core to the surface, and that heat illness will shortly ensue. In the present study, the mean difference between mean rectal temperature and mean skin temperature was exactly $1.0^{\circ} \mathrm{C}$ at the last minute of peak exercise in the Tborg runs, indicating impending heat stress. However, in the the T/cryo runs, the temperature difference between Tsk and Tre was a full $4.0^{\circ} \mathrm{C}$, and the A/cryo runs produced a temperature difference of $1.7^{\circ} \mathrm{C}$. The large gas flow, nearly $400 \mathrm{~V} / \mathrm{min}$, circulated through the suit by the cryogenic life support system, effectively cooled the subject so that heat stress was minimal. 


\section{Heart Rate}

Heart rates reached equilibrium very quickly in the $C$ runs, and remained relatively constant throughout the exercise period until the cool down. In the Tborg runs, the heart rate rose very quickly upon beginning the walking warm up and continued to rise throughout the test, never reaching steady state. This pheriomenon was also observed by other researchers $(7,12)$, although heart rates in Smolander's suited runs were lower than those observed in this study. Although heart rates in the T/cryo and $\mathrm{A} /$ cryo runs in our study were slightly higher at all levels compared to the same levels during the $C$ runs, the trend toward equilibrium was also seen in the $\mathrm{T} / \mathrm{cryo}$ and $\mathrm{A} / \mathrm{cryo}$ runs.

Cardiac stress was evident in the high heart rate response in the Tborg runs. Introduction of cooling in the T/cryo runs significantly decreased $(p=.00002)$ the heart rate and presumably the cardiac stress. Although most subject's heart rates remained low enough during the Tborg runs that no concern arose, at least three subjects were working at over $85 \%$ of their maximum heart rate at the last minute of exercise. These tests would have been terminated due to safety concerns if the trend had continued for a few more minutes. This is a further indication that the subject's exercise or working time while wearing the Tborg ensemble was limited by physiological stress. The convective cooling provided in the T/cryo ensemble kept the majority of the subjects under $100 \mathrm{bpm}$ throughout the test run. This would equal to only $55 \%$ of the test subjects predicted maximal heart rate based on the mean ages of all subjects. The mean heart rate of $121 \mathrm{bpm}$ during the A/cryo runs at the last minute of peak exercise was also significantly different than in the 
Tborg runs ( $p=.03$ ).

\section{Body Water Loss}

The average sweat loss measured during the Tborg tests in this study was nearly four times what was measured in the unsuited control tests. This is consistent with data found in the literature in similar enclosed suits $(11,12)$. With the introduction of cooling, there was a dramatic decrease in body water loss, from $0.96 \mathrm{~kg}$ in the Tborg runs to only $0.38 \mathrm{~kg}$ in the T/cryo runs, and $0.47 \mathrm{~kg}$ in the A/cryo runs. Since the Ames prototype suit fit most subjects much more snugly than the standard HazMat suit, it is presumed that the increased sweat rate was due to lack of cooled air reaching the limbs, and therefore less of an opportunity for evaporative coóling.

The Fire Department to which the test subjects in this study belonged has body water loss standards that must be adhered to during Level A HazMat entries [15]. According to these standards, body weight loss must not exceed $1.5 \%$ of total body weight. In a situation in which a $1.5 \%$ body water loss occurs, the HazMat worker is not allowed to reenter the scene. One subject in this study lost just over $1.5 \%$ of his body weight in the Tborg run, and two other subjects came within several tenths of a kilogram of the $1.5 \%$ limit. No subject lost more than $0.05 \%$ of his body weight in the T/cryo runs and no more than $0.07 \%$ of total body weight with the $\mathrm{A} / \mathrm{cryo}$ runs. Although it should be noted that the largest and heaviest subjects did not perform the A/cryo runs due to the size limitations of the prototype suit. These sweat loss data also clearly show that the cryogenic backpack had a very positive effect on the level of thermal strain experienced by the test 
subjects.

Ratings of Perceived Exertion and Thermal Comfort

As would be expected from the quantitative data already presented, the highest (i.e., warmest) ratings of percieved thermal comfort were given by subjects when performing the Tborg run. Interestingly, the average thermal comfort rating at the 40 min mark of Stage 3 in the T/cryo runs was 4.2, which was lower than the average rating of 4.6 in the $C$ run. The thermal comfort ratings at 5 minutes into Stage 3 in the T/cryo runs were also cooler than at 5 minutes into Stage 3 in the C runs, therefore indicating the subjects were cooler at the very start of the protocol than they were in shirt sleeves in the $24.4^{\circ} \mathrm{C}$ ambient lab environment.

Perceived exertion was also positively effected by the cryogenic cooling. Although no subject felt that the work ever got harder than " 7 " ( 1 subject), the highest average perceived exertion ratings were given at the $40 \mathrm{~min}$ mark in Stage 3 of the Tborg runs and at the same point in the A/cryo runs. Subjects noted in the $\mathrm{A} /$ cryo runs that the encumberance of the prototype suit and the tightness in the chest caused by the life support harness were reasons they gave a higher rating of perceived exertion. Also, the prototype suit did not provide as adequate cooling of the extremities as the standard HazMat suit, therefore the increased heat build-up in the Ames prototype suit could have been another reason for higher perceived exertion ratings.

Another possible reason why the perceived exertion rates were higher in the Tborg runs was that subjects were constantly rubbing their heads against the visor to wipe the condensation off their face shield to improve visibility. Subjects also 
had to occasionally lift the head cavity of the garment up and off of their face to reposition the face shield for optimal visibility. This constant repositioning and tugging at the suit was a problem that many subjects commented on and is a common complaint during actual HazMat operations. The gas circulation and cooling eliminated all face shield fogging in the T/cryo runs, although the manipulation of the head cavity was still a problem. Subjects had positive comments about the hemispherical helmet in the Ames prototype suit in that it didn't fog up, didn't need constant repositioning and offered a much greater field of vision. They feit that this was a desirable design feature for future suits.

\section{Conclusions}

The results of this study have answered our primary research question. It is very clear that the convective cooling produced by using a cryogenic, liquid air backpack with a representative, current Level A HazMat suit, significantly decreased the thermal and cardiovascular stress, as well as the overall perception of exertion, of actual HazMat team members exercising at a level comparable to that in real HazMat situations. This was also true for the prototype HazMat suit developed by NASA.

The measured reduction in physiological and perceived stress should improve a HazMat worker's productivity and safety. For example, the level of fatigue produced by performing a given set of tasks in a fixed time will be greatly reduced. In addition, the available work time on site while wearing a HazMat ensemble could be significantly increased with the adoption of a life support system that provides cooling. 
The secondary research question was to compare the performance of the prototype HazMat suit developed by NASA with that of a suit representative of current HazMat suit technology. The results are less clear for this question.

The various physiological indices measured consistently show that the NASA suit (A/cryo) does not perform as well as the Trelleborg suit ( $T / c r y o$ ) when both are used with the liquid air ECU. However, as previously discussed, the current, first generation NASA suit incorporates a number of design features that were actually a handicap for these tests. A second generation suit that eliminated these unnecessary features would weigh significantly less (approximately the same as the standard HazMat suit). We believe that the measured performance deficit is actually caused primarily by the excessive weight of the current NASA design. We believe that the comparative physiological results of the $\mathrm{A} / \mathrm{cryo}$ and $\mathrm{T} / \mathrm{cryo}$ ensembles might be quite different for a second generation NASA suit.

The NASA suit was deliberately designed with very rudimentary mobility joints to enable adjustments in size to better fit an individual user. Both features are used in current spacesuits. This design feature was expected to significantly improve comfort and mobility. However only one suit was available, and it was too small for most of our subjects. The subjects commented very favorably on features such as the hemispherical helmet and the lack of a breathing mask and the quick disconnects that allowed the boots and gloves to be quickly removed or replaced. However, definitive answers regarding the benefits of these design features are not currently available. A second generation NASA suit using our evaluation and test results should be designed and tested. 


\section{Acknowledgements}

The authors would like to thank the following individuals for their help:

Curtis Lomax for his assistance in developing our data acquisition system,

Dr. Leslie Montgomery for his help and advice in statistical treatment of

the data, Jerry James and Phil Culbertson for the prototype suit, and Karen Bunn for her editorial expertise in producing the final manuscript.

- Special thanks must be given to Chief Doug Sporleder, Asst. Chief Ben Lopes, and Captain Kelly Seitz of the Santa Clara County (CA) Central Fire Protection District for their cooperation and support in providing us with test subjects, as well as the HazMat specialists at Central Fire for their willingness to participate in the study. 


\section{REFERENCE LIST}

1. White M, Hodous T,Vercruyssen M: Effects of thermal environment and chemical protective clothing on work tolerance, physiological responses, and subjective ratings. Ergonomics 1991;34(4):445-457

2. Patton J, Bidwell T, Murphy M, et al: Energy cost of wearing chemical protective clothing during progressive treadmill walking. Avi Space, and Environ Med 1995; 66:238-42.

3. Raven P, Davis T, Shafer C, et al: Maximal stress test performance while wearing a self-contained breathing apparatus. J Occup Med 1977;19: 802806.

4. Louhevaara V,Tuomi T, Korhonen $\mathrm{O}$, et al: Cardiorespiratory effects of respiratory protective devices during exercise in well-trained men. Eur J Appl Physiol 1984; 52:340-345.

5. James R, Dukes-Dobos F, Smith R. Effects of respirators under heat/work con ditions. Am Indust Hygiene Assoc 1984; 45: 399-404.

6. Raven P, Dodson A, Davis T. The physiological consequences of wearing industrial respirators: a review. Am Indust Hygiene Assoc 1979; 40: $517-$ 534.

7. White $M$, Vercruyssen $M$, Hodous $T$. Work tolerance and subjective responses to wearing protective clothing and respirators during physical work. Ergonomics 1989; 32(9): 111-1123. 
8. Teitlebaum A, Goldman R. Increased energy cost with multiple clothing layers. J Appl Physiol 1972; 32(6): 743-744.

9. Hardy J, DuBois E. The technic of measuring radiaiton and convection. J Nutr., 1938, 15:461-475.

10. Benjamin F. Compendium of human responses to the aerospace environment, Vol 1, NASA CR 1205 (1).

11. McLellan T. Heat strain while wearing the current Canadian or a new hotweather French NBC protective clothing ensemble. Aviat Space Environ Med 1996; 67: 1057-62.

12. Smolander J, Louhevaara V, Tuomi T, et al. Cardiorespiratory and thermal effects of wearing gas protective clothing. Int Arch Occup Environ Health 1984; 54: 261-270.

13. Webbon B, Montgomery L, Miller L, et al. A comparison of three liquidventilation cooling garments during treadmill exercise. Aviat Space Environ Med 1981; 52(7): 408-415.

14. Duncan H, Gardner G, Barnard R. Physiological responses of men working in fire fighting equipment in the heat. Ergonomics 1979; 22(5): 521-527.

15. California Specialized Training Institute Curriculum for HazMat Technicians and Specialists, Student Manual, Chapter 3 14-29, 1995.

16. Iampietro $P$, Goldman $R$. Tolerance of men working in hot, humid environments. J Appl Physiol 1965; 20: 73-76.

17. Borg G. Psychological bases of physical exertion. Med Sci Sports and Ex 1982; 14:377:381.

18. Young A, Sawka M, Epstein Y, et al. Cooling different body surfaces during 
upper and lower body exercise. J Appl Physiol 1987; 63(3): 1218-1223. 
Table 1. Exercise Protocol

Stage 1: Min 0-5 Standing control

Stage 2: Min 6-8 Warm up walk at $1.6 \mathrm{mph}$

Stage 3: Min 9-54 Walking at $2.0 \mathrm{mph}$

Stage 4: Min 55-57 Cool down walk at $1.6 \mathrm{mph}$

Stage 5: $\quad$ Min 58-62 Standing control 
Table 2. Perceived Exertion Scale (17)

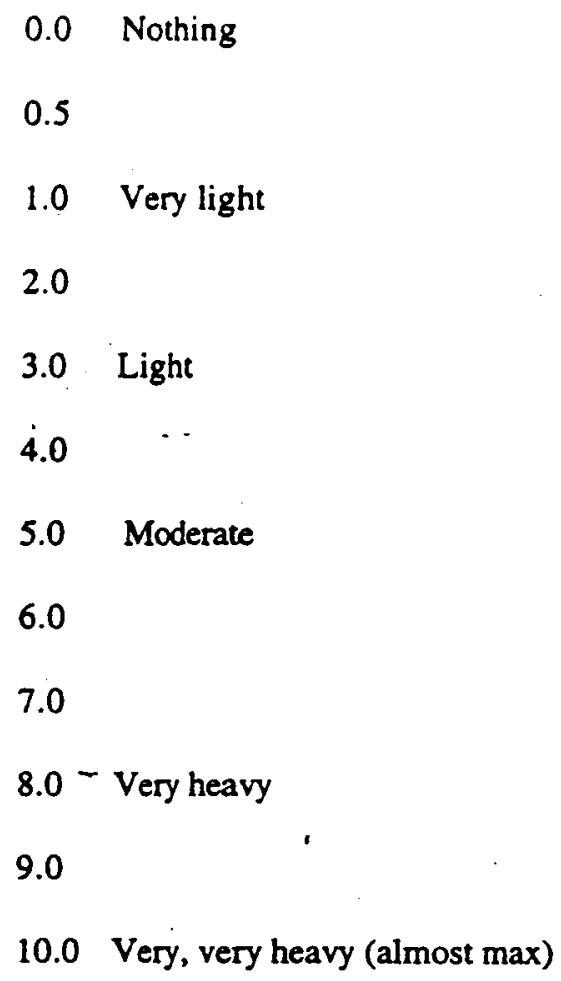


Table 3. Thermal Comfort Scale (18)

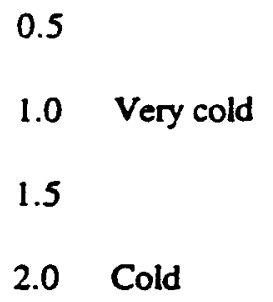

8.0 Unbearably hot 
2

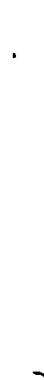




\section{Figure Legends}

\section{1.}

Mean oxygen consumption during the control period $\square$ and at the last minute of exercise for unsuited control and the three suit configurations: Trelleborg with SCBA (Tborg), Trelleborg with Cryogenic Environmental Control Unit (T/cryo), and the Ames suit with cryogenic ECU (A/cryo).

2.

Mean core temperature during the control period $\square$ and at the last minute of exercise unsuited control and the three suit configurations.

3.

Mean body temperature during control period unsuited control and the three suit configurations.

4.

Mean body temperature during control period $\because$ and at the last minute of exercise $\mathbb{Z}$ for unsuited control and the three suit configurations.

5.

Sweat losses for unsuited control and the three suit configurations.

6.

Average exertion ratings at 5 minutes into Stage $3 \square$ and at 40 minutes into Stage $3 \mathbb{C}$. 7.

Average thermal comfort ratings at 5 minutes into Stage 3 and at 40 minutes into Stage 3 


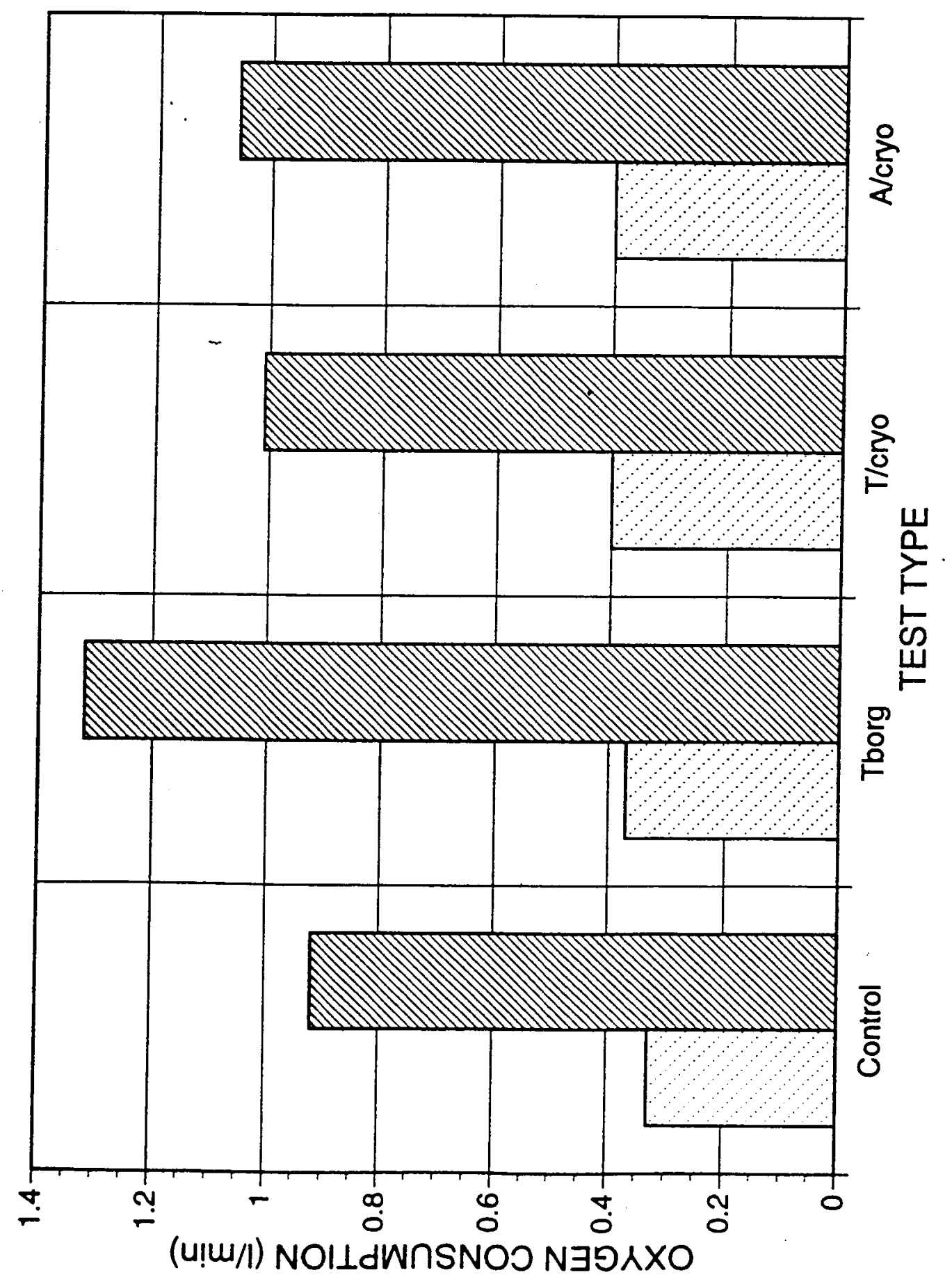




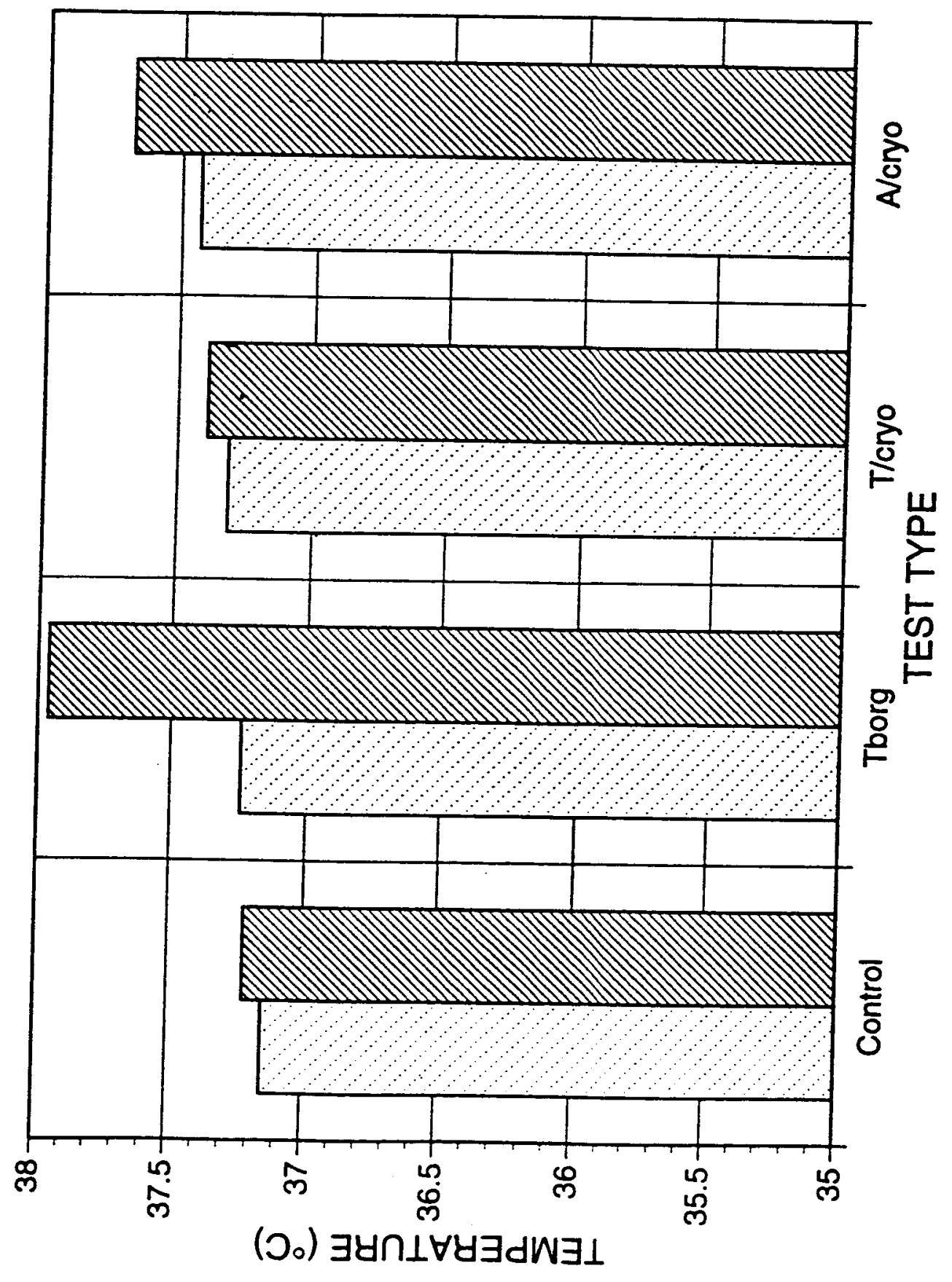




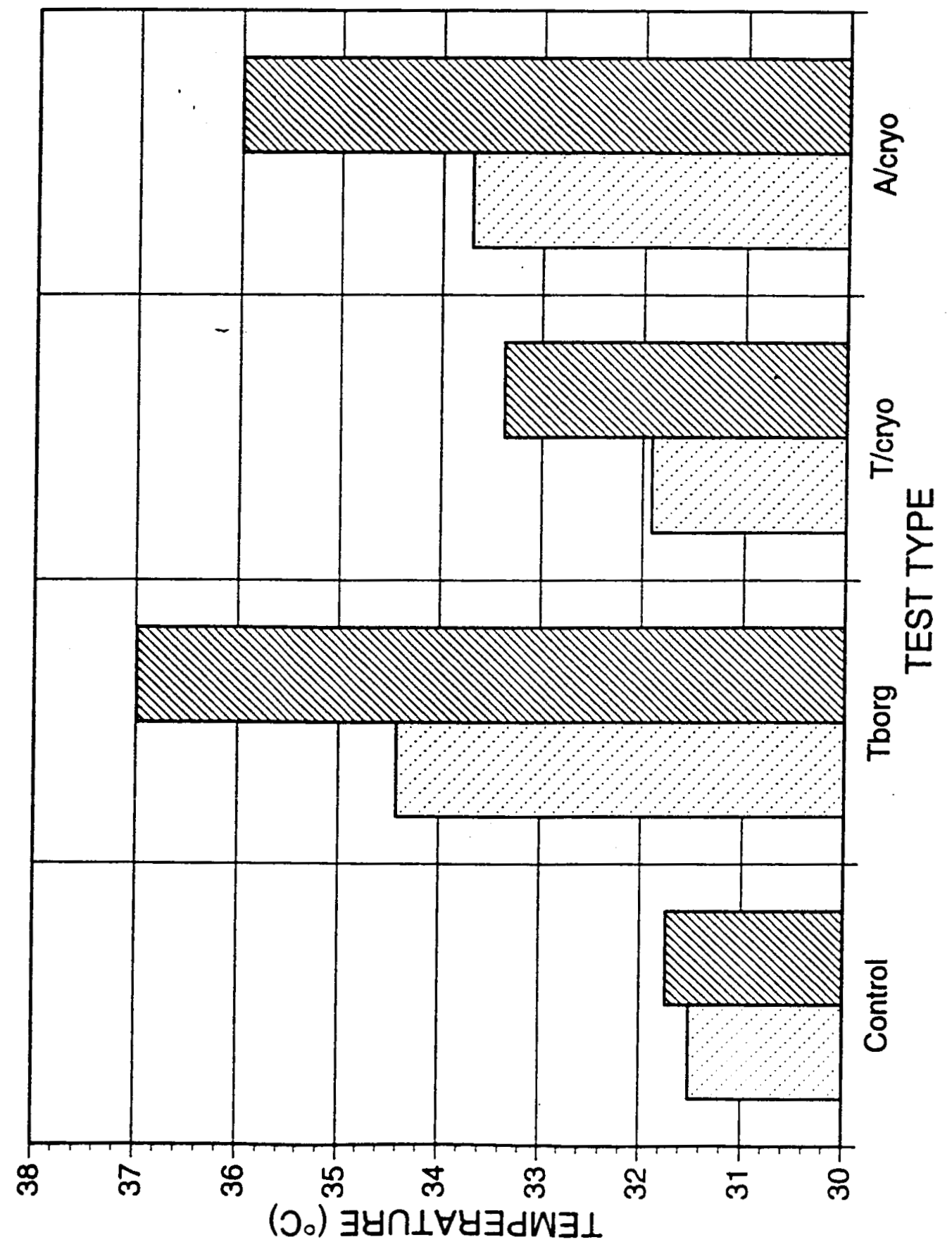




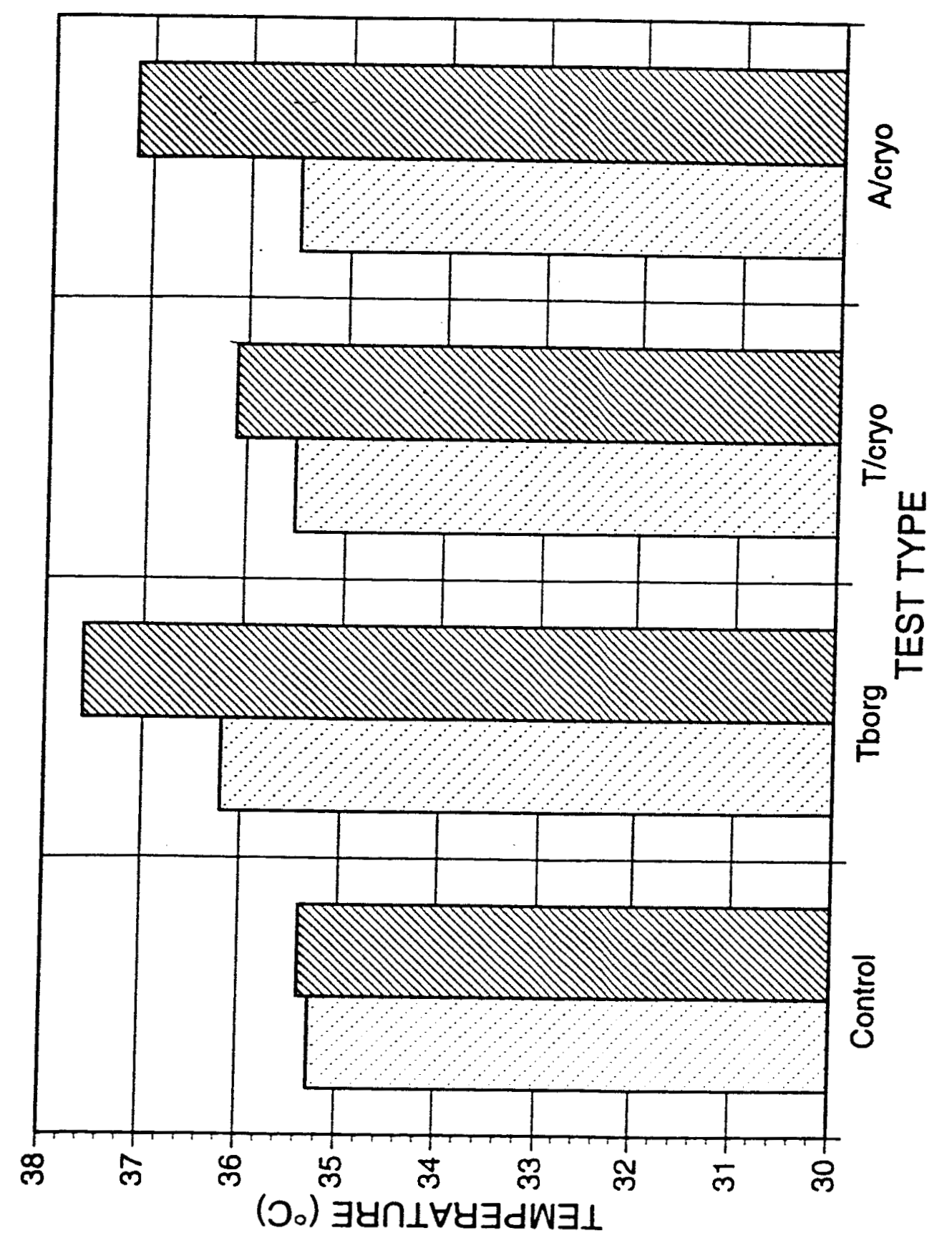




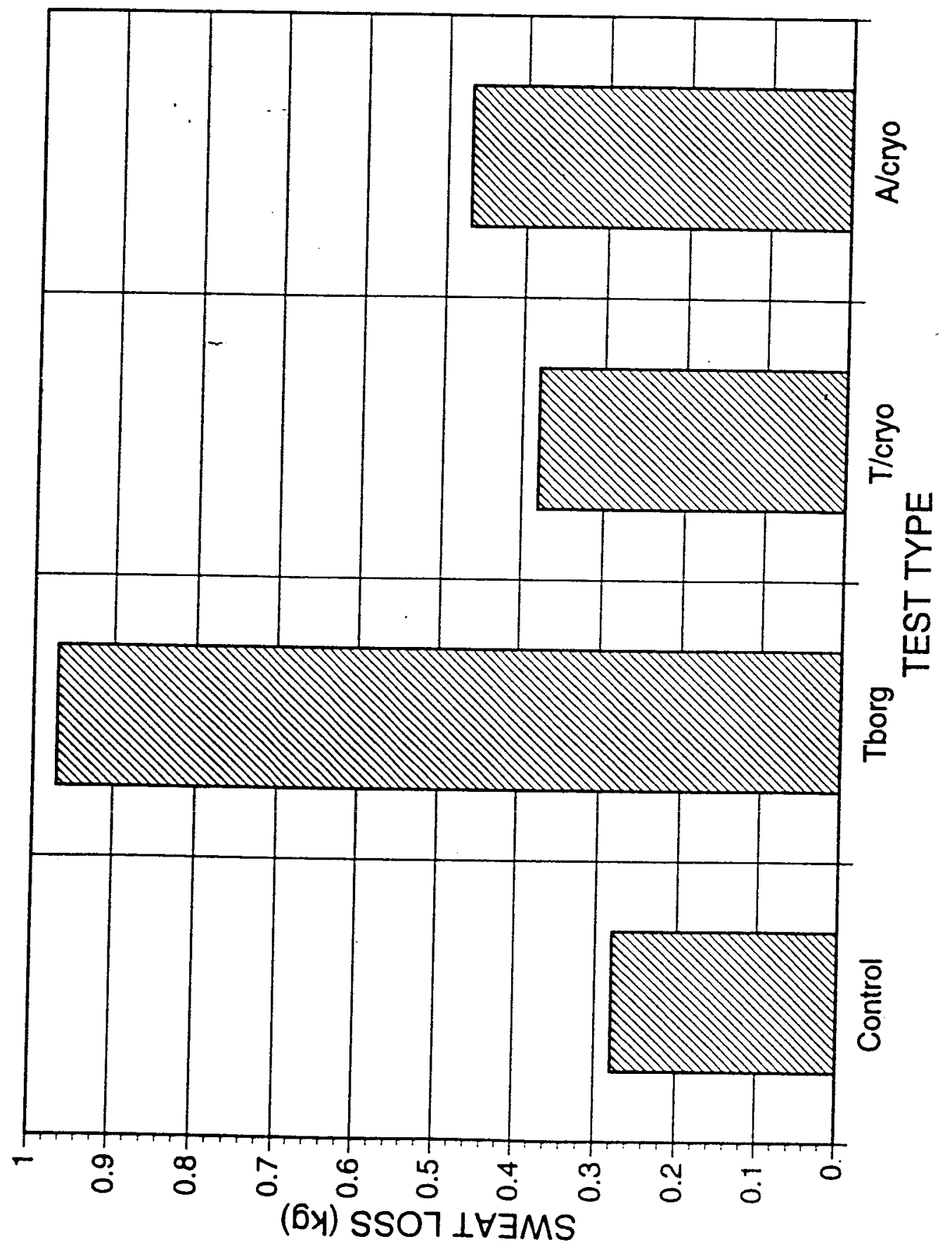




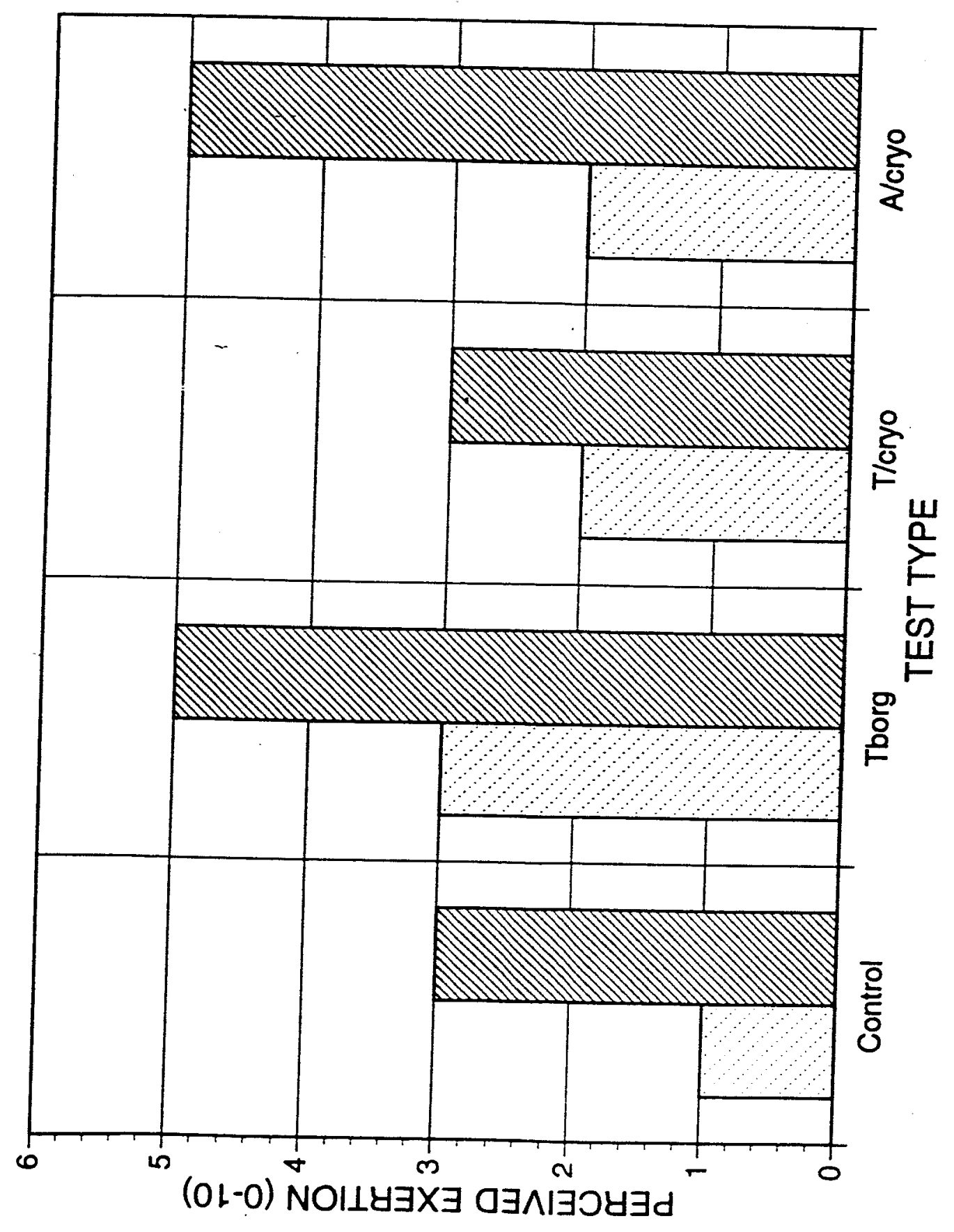




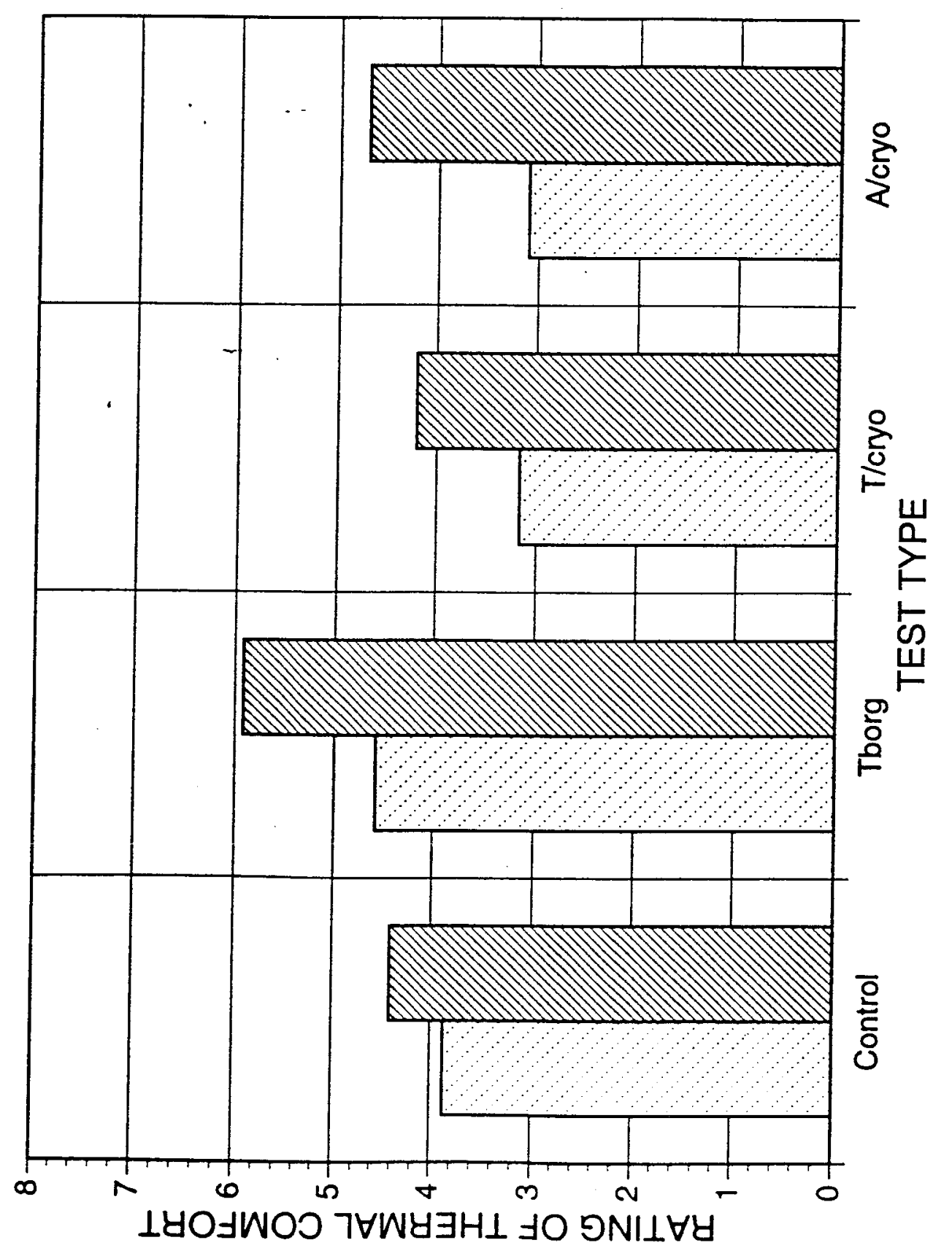

